\title{
ASCA Observations of Seyfert 2 Galaxies
}

\author{
H. Awaki \\ Department of Physics, Faculty of Science, Kyoto University, \\ Kitashirakawa, Sakyo, Kyoto, 606-01, Japan
}

\begin{abstract}
We have observed a sample of Seyfert 2 galaxies with $A S C A$. The targets were selected based on their [O III] $\lambda 5007 \mathrm{flux}$, the existence of polarized broad lines, and previous X-ray detection. The nuclear luminosities of these galaxies range from $10^{42}$ to $10^{44} \mathrm{ergs} \mathrm{s}^{-1}$ in the $2-10 \mathrm{keV}$ band. Obscured nuclei were found in all galaxies in our sample, with absorption column densities distributed around a value of $10^{23.5} \mathrm{~cm}^{-2}$.

The cold iron $\mathrm{K} \alpha$ line was clearly detected in half of our sample, and emission from He-like and $\mathrm{H}$-like $\mathrm{Fe} \mathrm{K} \alpha$ was detected from two galaxies. These lines are explained by fluorescent lines from thick cold matter and warm/hot thin matter. Highly ionized lines originating in starburst activity were also detected from NGC 1068.
\end{abstract}

\section{Introduction}

It is well known that Seyfert 2 galaxies have high-ionization lines, no broad lines, and weak activity compared with Seyfert 1 galaxies. Spectropolarimetry led to the detection of polarized broad lines in several Seyfert 2 galaxies (e.g., Antonucci \& Miller 1985). These observations suggest obscuration of the central region. Antonucci \& Miller proposed a torus model in which direct optical light is completely blocked by thick matter and thus only scattered light is observed. If we use a sufficiently high-transmission band, however, the central region can be seen through the torus. Hard $\mathrm{X}$-rays above $3 \mathrm{keV}$ can penetrate thick matter with column densities up to $10^{23} \mathrm{~cm}^{-2}$. Thus X-ray observations provide a means of obtaining information about Seyfert 2 nuclei.

The Japanese X-ray satellite GINGA detected hard X-rays from a number of Seyfert 2 galaxies (e.g., Awaki et al. 1991). This result is explained naturally by the torus model. However it is still unclear whether all Seyfert 2 galaxies have luminous nuclei. The Japanese satellite $A S C A$ has the best sensitivity to date in the $2-10 \mathrm{keV}$ band; we can obtain an $\mathrm{X}$-ray spectrum of a source with the 2-10 keV flux of a few $\times 10^{-13} \mathrm{ergs} \mathrm{s}^{-1} \mathrm{~cm}^{-2}$ in a one-day exposure, and hence can observe most of Seyfert 2 galaxies with $L_{\mathrm{x}}>10^{45} z^{2} \mathrm{ergs} \mathrm{s}^{-1}$, where $L_{\mathbf{x}}$ and $z$ are the X-ray luminosity and redshift, respectively. We assume $H_{0}=50 \mathrm{~km} \mathrm{~s}^{-1} \mathrm{Mpc}^{-1}$ throughout this discussion. 


\section{X-Ray Characteristics of Seyfert 2 Galaxies}

\subsection{Target List}

We selected a sample of Seyfert 2 galaxies by the following criteria: (1) prominent [O III] $\lambda 5007$ line emission, (2) polarized broad lines, and (3) previous X-ray observations. The targets are listed in Table 1. The 'bright [O III] sample' sources were drawn from Dahari \& De Robertis (1988) and Whittle (1992). The first nine targets in the Table (NGC 1068 to NGC 5643) are among the 10 brightest galaxies in $[\mathrm{O}$ III $] \lambda 5007$. The tenth galaxy in the list, NGC 1386 , has been observed with ASCA (Makishima et al. 1997). These constitute an unbiased X-ray sample. On the other hand, polarized broad lines (PBL) have been discovered in ten Seyfert 2 galaxies, of which we have observed eight. Including NGC 7672, which is a GINGA source, nine PBL Seyfert 2 galaxies have been observed in the $\mathrm{X}$-ray band.

Table 1. Target List

\begin{tabular}{lrrrl}
\hline \hline Target Name & Redshift & $f([\mathrm{O} I \mathrm{II}])^{\mathrm{a}}$ & PBL $^{\mathrm{b}}$ & X-ray reference \\
\hline NGC 1068 & 0.0038 & 2100 & AM85 & Ueno et al. 1994 \\
Mrk 3 & 0.0135 & 440 & MG90 & Iwasawa et al. 1994 \\
Mrk 477 & 0.0378 & 220 & T92 & Ueno et al. 1996a \\
1319-164 & 0.0172 & 150 & & Ueno et al. 1996a \\
NGC 3081 & 0.0080 & 125 & & Awaki \& Griffiths \\
& & & & \\
NGC 4507 & 0.0117 & 110 & & Ueno et al. 1996a \\
Mrk 1210 & 0.0135 & 100 & T92 & Ueno et al. 1996a \\
IC 5063 & 0.0113 & 93 & & Ueno et al. 1996a \\
NGC 5643 & 0.0040 & 80 & & Awaki \& Matsuoka \\
NGC 1386 & 0.0031 & 79 & & Makishima et al. 1997 \\
& & & & \\
Mrk 463E & 0.0497 & 73 & MG90 & Ueno et al. 1996b \\
NGC 4388 & 0.0084 & 66 & & Iwasawa et al. 1996 \\
Was 49b & 0.0630 & $66 ?$ & T92 & Ueno et al. 1996a \\
NGC 7212 & 0.0260 & 57 & T92 & Ueno et al. 1996a \\
Mrk 348 & 0.0149 & 23 & MG90 & Weaver \& Awaki \\
NGC 7172 & 0.0086 & & & Svensson \& Ueno \\
NGC 7319 & 0.0225 & 6.4 & & Awaki et al. 1996 \\
\hline
\end{tabular}

${ }^{\mathrm{a}}$ In units of $10^{-14} \mathrm{ergs} \mathrm{s}^{-1} \mathrm{~cm}^{-2}$.

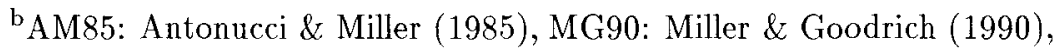
T92: Tran et al. (1992). 

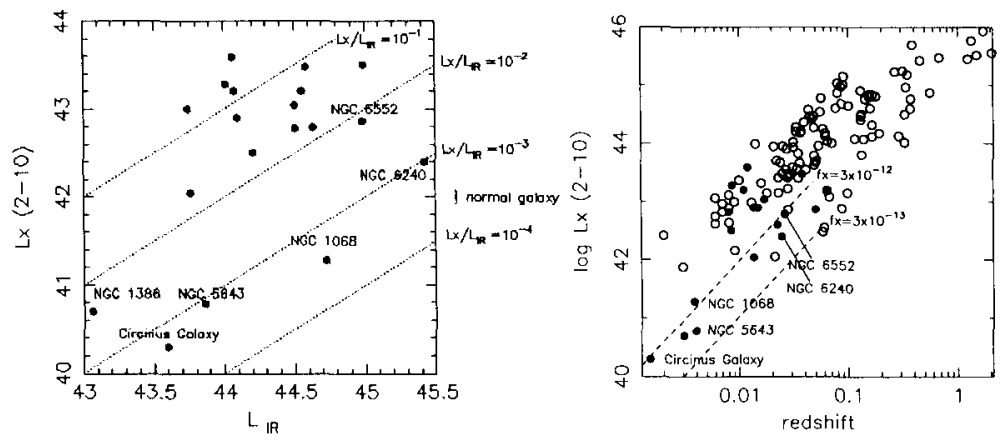

Figure 1. Left: $L_{\mathrm{x}}$ vs. $L_{\mathrm{IR}}$. Right: Comparison of $L_{\mathrm{x}}$ for Seyfert 1 and 2 galaxies. Open and closed circles display Seyfert 1 and 2 galaxies, respectively.

\subsection{Results}

We obtained X-ray spectra of all the galaxies in our sample. The spectra are not well-described by a single-component model (e.g., a power-law continuum). Therefore, we fitted the spectra with a two-component model consisting of thin thermal emission (or power-law continuum) and an absorbed power-law continuum. We also included an emission line around $6.4 \mathrm{keV}$. This model works well for all of our spectra.

While the observed spectra require an absorbed power-law continuum, the existence of hard X-ray emission alone is not sufficient evidence for AGN activity because normal galaxies also have hard $\mathrm{X}$-ray emission which correlates well with the far-IR luminosity $\left(L_{\mathbf{x}} / L_{\mathrm{IR}}=10^{-3}-10^{-4}\right)$. We first examined AGN activity using this correlation. Figure 1 (left) shows a $L_{\mathrm{x}}-L_{\mathrm{IR}}$ diagram for our sample. We found that most of the galaxies in our sample have excess emission above the correlation line for normal galaxies, and that their luminosities range from $10^{42}$ to $10^{44} \mathrm{ergs} \mathrm{s}^{-1}$ in the $2-10 \mathrm{keV}$ band. For NGC 1068 and NGC 5643, we found strong iron lines in their X-ray spectra, suggesting that the direct beam is completely blocked by thick matter. Thus, we concluded that these galaxies do indeed have $A G N$ activity.

Next, we compared levels of activity for Seyfert 2 and Seyfert 1 galaxies. Figure 1 (right) shows the luminosity for both types of Seyfert galaxies. No clear difference between Seyfert 1 and Seyfert 2 galaxies is found. Therefore, our data imply that Seyfert 2 galaxies have the same level of activity as Seyfert 1 galaxies.

Since we selected the data based on the existence of polarized broad lines and prominent [O III] lines, our results indicate that Seyfert 2 galaxies with polarized broad lines have luminous nuclei as well as broad-line regions. We found luminous nuclei in the bright-[O III] sample. The luminosity of the [O III] line ranged from $5 \times 10^{40}$ to $10^{43} \mathrm{ergs} \mathrm{s}^{-1}$; most Seyfert 2 nuclei are in this range. Therefore, we conclude that Seyfert 2 galaxies with prominent [O III] lines show 


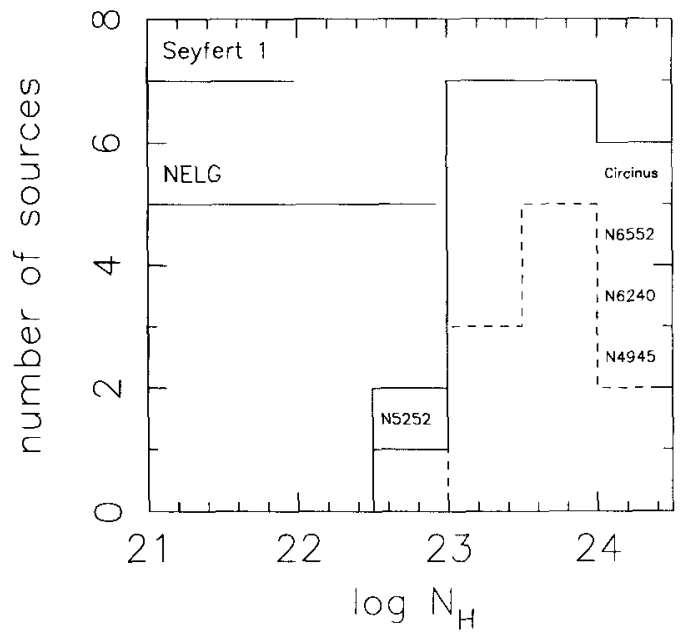

Figure 2. The distribution of absorbing column. The targets with $N_{\mathrm{H}}>10^{24} \mathrm{~cm}^{-2}$ are located at $\log N_{\mathrm{H}}=24.0-24.5$. The dashed line shows the column density for the brightest sample. The data for NGC 4945, NGC 5252, NGC 6240, NGC 6552, and Circinus galaxy are from Iwasawa (1994), Cappi et al. (1996), Nakagawa et al. (1996), Fukazawa et al. (1994), and Matt et al. (1996), respectively.

AGN activity, and a prominent [O III] line is a suitable criterion for identification of Seyfert 2 galaxies.

As noted above, each galaxy spectrum requires an absorbed power-law component. Figure 2 shows the distribution of the absorbing column $\left(N_{\mathrm{H}}\right)$. Since ASCA cannot measure column densities greater than $10^{24} \mathrm{~cm}^{-2}$, the targets with $N_{\mathrm{H}}>10^{24} \mathrm{~cm}^{-2}$ are included at $\log N_{\mathrm{H}}=24-24.5$. The dashed line shows the column density for the bright-[O III] sample. We added data for other available Seyfert 2 galaxies to our sample. For Seyfert 1 galaxies and narrow emission-line galaxies (NELG), the column densities were distributed below $10^{22} \mathrm{~cm}^{-2}$, and between $10^{21}$ and $10^{23} \mathrm{~cm}^{-2}$, respectively. The column densities for Seyfert 2 galaxies are 10-100 times larger than those of Seyfert 1 galaxies and NELGs.

One remarkable result is the concentration of the column densities around $10^{23.5} \mathrm{~cm}^{-2}$. There are only a few objects with $N_{\mathrm{H}}>10^{24} \mathrm{~cm}^{-2}$. Assuming a simple model based on a uniform-density torus, this may indicate that the shape of the obscuring matter is not disk-like, but donut-like. If it were disk-like, there would be many galaxies with high column densities.

\section{Line Emission from Seyfert 2 Galaxies}

\subsection{Line Emission from Cold Iron}

We clearly detected emission lines around $6.4 \mathrm{keV}$ from half of our targets. The energy of $6.4 \mathrm{keV}$ corresponds to the $\mathrm{Fe} \mathrm{K} \alpha$ line from cold matter. The lines from 


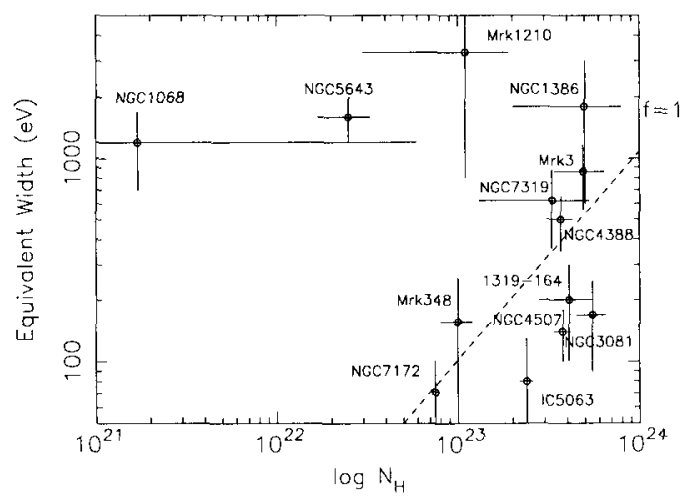

Figure 3. The column density vs. equivalent width ( $E W$ ) of the $\mathrm{Fe}$ $\mathrm{K} \alpha$ line. The dashed line shows the relation between $E W$ and $N_{\mathrm{H}}$ in the simplest model of isotropic radiation and uniformly distributed matter.

a few galaxies were broadened; however, narrow-line fits were also acceptable at the $90 \%$ confidence level. We assumed a narrow line in estimating the equivalent width of $\mathrm{Fe} \mathrm{K} \alpha$ line $(E W)$. Measured values of $E W$ range from $100 \mathrm{eV}$ to $2000 \mathrm{eV}$ (Fig. 3).

Since the continuum emission does not have a thermal origin, we take the Fe $\mathrm{K} \alpha$ line to be a fluorescence line. Taking account of scattered light emitted from cold matter, the $E W$ is

$$
E W=\frac{(\text { iron line })}{(\text { direct component })+(\text { scattered light })}
$$

where the direct and scattered-light components are measured at $6.4 \mathrm{keV}$. Assuming isotropic radiation, $E W \approx 100 f\left(N_{\mathrm{H}} / 10^{23}\right) \mathrm{eV}$ in cases where $N_{\mathrm{H}}<$ $10^{24} \mathrm{~cm}^{-2}$, where $f$ is the covering factor (e.g., Awaki et al. 1991). To produce $E W \approx 100 \mathrm{eV}$, the cold matter should be characterized by $N_{\mathrm{H}}>2 \times 10^{23} \mathrm{~cm}^{-2}$ and $f>0.5$. The range of $E W$ from 100 to $2000 \mathrm{eV}$ can be explained by differences in the covering factor, time variations, and/or different relative amounts of extinction of the direct component.

Extinction is especially important. If the direct beam is completely blocked, we see only reflected light and fluorescence lines from the thick matter. In this regard, the Circinus galaxy is an interesting object. The spectrum has a strong iron $\mathrm{K} \alpha$ line near $6.4 \mathrm{keV}$ (Fig. 4, left), and the spectrum above $3 \mathrm{keV}$ is well fitted by a Compton reflection model plus emission lines (Matt et al. 1996). This spectrum is surprisingly similar to the X-ray spectrum of Vela X-1 in eclipse, as shown in Fig. 4 (right) from Nagase et al. (1994). The direct beam of Vela X-1 is completely blocked by the primary star and only the reflection component is observed in the hard X-ray band. This similarity is strong evidence that the Circinus galaxy exhibits a reflection-dominated spectrum. Another $A S C A$ example of a reflection-dominated spectrum is NGC 6552 (Fukazawa et al. 1992). 

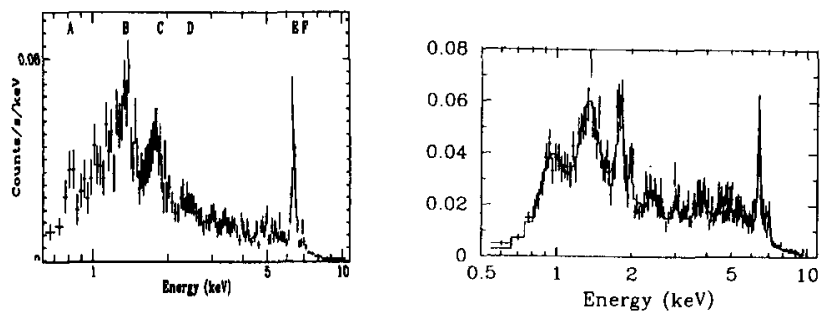

Figure 4. Left: The X-ray spectrum of the Circinus galaxy (Matt et al. 1996). Right: Vela X-1 in eclipse (Nagase et al. 1994).

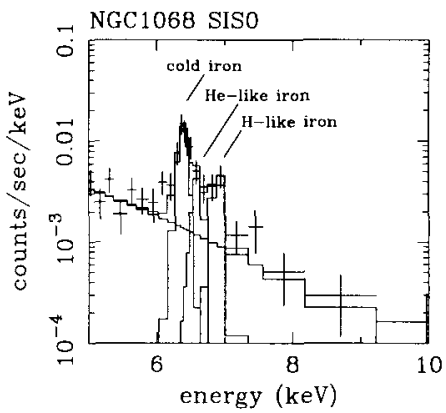

Figure 5. Fe line emission from NGC 1068 (Ueno, private communication).

\subsection{Line Emission from Warm Iron}

Two galaxies, NGC 1068 and NGC 6240 (Nakagawa et al. 1996), show He-like and $\mathrm{H}$-like $\mathrm{Fe} \mathrm{K} \alpha$ in their $\mathrm{X}$-ray spectra, as well as cold $\mathrm{Fe} \mathrm{K} \alpha$. Figure 5 shows a close view of the X-ray spectrum for NGC 1068. The emission lines from $\mathrm{He}$-like and $\mathrm{H}$-like iron were clearly detected. To produce $\mathrm{H}$-like iron, ionization parameters $\left(\xi=L / n r^{2}\right)$ must have values as large as 1000 , and the temperature of the photoionized plasma is about $10^{6} \mathrm{~K}$ (Kallman \& McCray 1982). Since we also detected cold iron, there may be a two-temperature plasma. Marshall et al. (1993) estimated the temperature of the plasma, and found $T=2 \times 10^{5} \mathrm{~K}$ for a warm component and $T=4 \times 10^{6} \mathrm{~K}$ for a hot component. Figure 6 is a schematic view of the scattering region by Marshall et al. (1993).

If this structure is common for Seyfert 2 galaxies, this scattering component contributes to the soft X-ray emission detected with $A S C A$. In fact, Ueno et al. (1996) pointed out that the soft component in several galaxies arises from AGN activity.

\subsection{Line Emission from a Thin Thermal Plasma}

For NGC 1068, Ueno et al. (1994) found a large number of emission lines from highly ionized ions in the low-energy band. The spectrum including these lines 


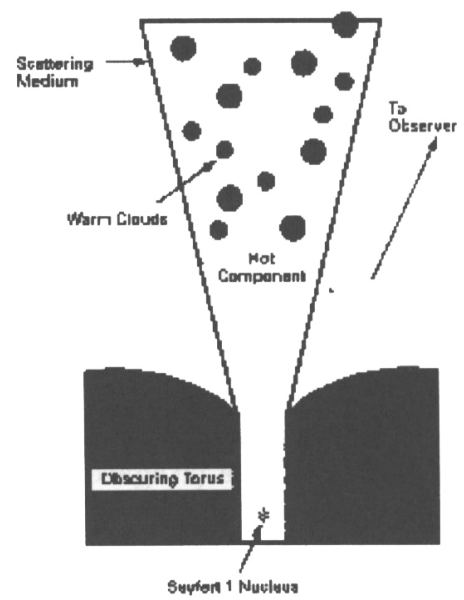

Figure 6. A schematic view of the scattering region (Marshall et al. 1993).

was well fitted by a two-temperature plasma, with $k T=0.59 \mathrm{keV}$ and $k T=$ $0.14 \mathrm{keV}$. These lines are thought to represent thermal emission associated with the starburst phenomenon (Ueno et al. 1994).

\section{Summary}

We found an obscured nucleus in every galaxy in our sample, which span a range of luminosity from $10^{42}$ to $10^{44} \mathrm{ergs} \mathrm{s}^{-1}$ in the $2-10 \mathrm{keV}$ band. We find that the absorbing column densities are distributed around $10^{23.5} \mathrm{~cm}^{-2}$.

The $6.4 \mathrm{keV}$ iron lines are clearly detected in half of our sample. This indicates the existence of cold thick matter. For two galaxies, He-like and $\mathrm{H}-$ like Fe lines, as well as the cold iron line, were detected, which indicates the existence of warm and hot matter. Highly ionized emission lines were found in the spectrum of NGC 1068. Considering that NGC 1068 has starburst activity, these emission lines could originate from thin thermal plasma associated with the starburst phenomenon.

Figure 7 shows a schematically how these results apply to the torus model.

Acknowledgments. The author thanks Professor Koyama, Dr. Ueno, and many other members of the ASCA team for their help to this work. This work was supported by the Scientific Research Fund of the Ministry of Education, Science, and Culture under Grant No. 07454040.

\section{References}

Antonucci, R. R. J., \& Miller, J. S. 1985, ApJ, 297, 621.

Awaki, H., et al. 1991, PASJ, 43, 195.

Awaki, H., et al. 1996, in preparation. 


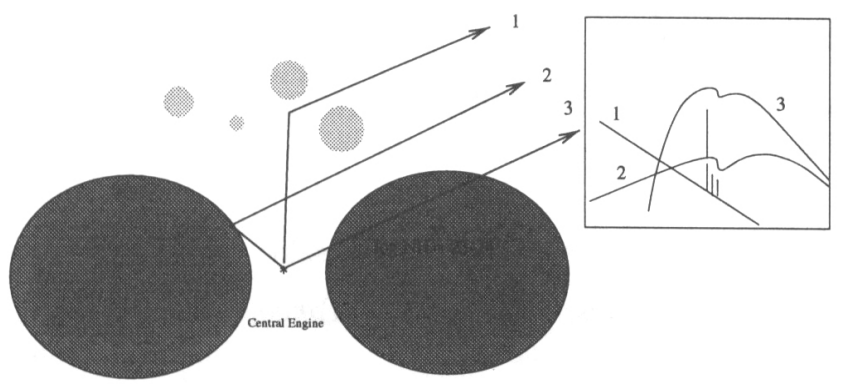

Figure 7. A schematic view of a Seyfert 2 galaxy and X-ray emission components: (1) scattered light by warm and hot plasma, (2) reflected light by cold thick matter (torus), and (3) direct light from a central engine through the torus.

Cappi, M., et al. 1996, ApJ, 456, 141.

Dahari, O., \& De Robertis, M. M. 1988, ApJS, 67, 249.

Fukazawa, H., et al. 1994, PASJ, 46, L141.

Iwasawa, K., et al. 1994, PASJ, 46, L167.

Iwasawa, K. 1994, Ph.D. Thesis, Nagoya University.

Iwasawa, K., et al. 1996, to be submitted to A\&A.

Kallman, T.R., and McCray, R. 1982, ApJS, 51, 263.

Makishima, K., et al. 1997, this volume.

Marshall, F., et al. 1993, ApJ, 405, 168.

Matt, G., et al. 1996, MNRAS, in press.

Miller, J. S., and Goodrich, R.W. 1994, ApJ, 355, 456.

Nagase, F., et al. 1994, ApJ, 436, L1.

Nakagawa, T., et al. 1996, in preparation.

Tran, H. D., et al. 1992, ApJ, 397, 452.

Ueno, S., et al. 1994, PASJ, 46, L71.

Ueno, S., et al. 1996a, in preparation.

Ueno, S., et al. 1996b, PASJ, 48, 389.

Whittle, M. 1992, ApJS, 79, 49. 